\title{
SCIIENCE

\section{Anais do \\ I Colóquio da Pós-Graduação do IF Goiano - Campus Urutaí}

IV SIMPÓSIO EM PROTEÇÃO DE PLANTAS

II SEMINÁRIO DE PESQUISA EM EDUCAÇÃO BÁSICA

\section{WORKSHOP DE RECURSOS NATURAIS DO CERRADO}

\author{
Áreas Temáticas: \\ Conservação de Recursos Naturais do Cerrado \\ Ensino para Educação Básica \\ Proteção de Plantas
}

Palestras - Mesa Redonda - Apresentação de Trabalhos

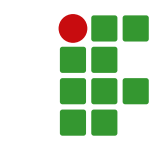

INSTITUTO

FEDERAL

Goiano

Campus
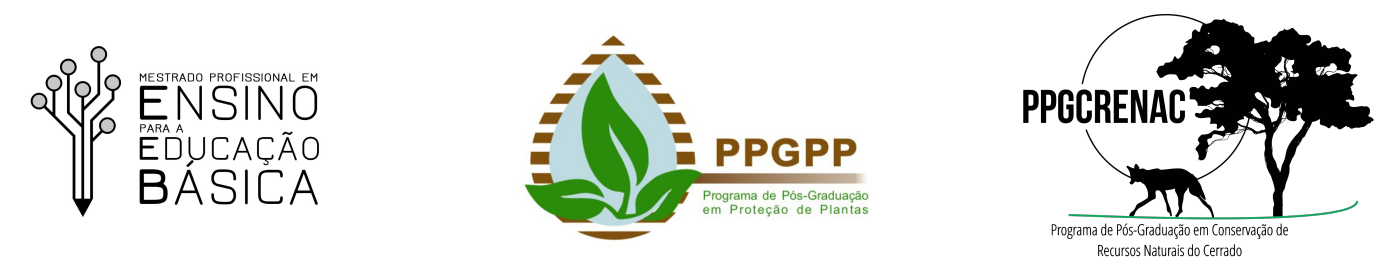


\section{Nota sobre o I CoPU}

A Pós-Graduação Profissional é um segmento cada vez mais relevante quando se trata de capacitação em pesquisa aplicada. É considerando isso que a Pós-Graduação do Instituto Federal Goiano - Campus Urutaí promoveu nos dias 4 e 5 de novembro de 2020 o I Colóquio da Pós-Graduação do IF Goiano Campus Urutaí, evento cunhado sobre a premissa do compartilhamento de experiências e soluções, técnico-científicas ou de gestão, por docentes, discentes e profissionais de diferentes áreas do conhecimento.

Esta primeira edição ocorreu de forma totalmente virtual e gratuita, com atividades específicas e amplas no âmbito dos Programas de Pós-Graduação em Proteção de Plantas, Conservação de Recursos Naturais do Cerrado, e Ensino para a Educação Básica. Estes PPGs realizaram em paralelo seus eventos anuais de base técnico-científica.

O IV Simpósio em Proteção de Plantas trouxe, mais uma vez, integração e atualização de conteúdos técnico-científicos aplicados, principalmente, ao manejo de insetos-pragas, doenças, nematóides e plantas daninhas. Nesta quarta edição, o público alvo - produtores rurais, estudantes dos cursos técnico, superior e pós-graduação e docentes - teve a oportunidade de verificar alternativas tecnológicas e de inovação em Fitossanidade.

O V Workshop de Conservação de Recursos Naturais do Cerrado permitiu que os participantes tivessem renovadas reflexões sobre atitudes e práticas que minimizem a degradação do Cerrado. Nesta edição do evento houve discussões acerca da ciência e a formação de cientistas no Brasil.

O II Seminário de Pesquisa em Educação Básica promoveu a divulgação de estudos desenvolvidos para melhoria nos processos de ensino na Educação Básica brasileira, por meio de trabalhos conduzidos por discentes, docentes e comunidade externa. Os inscritos puderam assistir palestras de pesquisadores renomados da área de Ensino e participaram de discussões que envolvem a formação continuada de professores, ensino para a cidadania e tecnologias educacionais.

Entre 23/09 e 23/10 a Comissão Científica do I CoPU selecionou resumos simples e trabalhos completos para serem apresentados. Os resumos vieram acompanhados de abstract e link para vídeo de apresentação dos trabalhos. Estes estão disponíveis nos Canais dos PPGs no YouTube. Multi-Science Journal não poderia deixar de prestigiar a divulgação destes resumos em sua edição v. 3, n. 3 (2020), lhes atribuindo DOI gratuitamente. Os trabalhos completos foram publicados em anais específicos para tal, recebendo ISBN, e estarão disponíveis na página do evento.

Os selecionados para apresentação virtual ao vivo foram avaliados por docentes convidados dos PPGs. No encerramento do evento houve premiação com certificado de honra ao mérito para o melhor trabalho.

\section{Links úteis:}

- Página do evento: http://bit.ly/ICoPU

- Canal da Diretoria de Pesquisa no YouTube: https://www.youtube.com/channel/UCjca_97wjGgr6VPhkRJHoQ/

- Canal do PPGPP: https://www.youtube.com/channel/UCWBj9Tn-rFtuXjKbToGkrnA

- Canal do PPG-CRENAC: https://www.youtube.com/channel/UC_pv_NMXYoktG2iRzeffqww

- Canal do PPG-ENEB: https://www.youtube.com/channel/UCL0nepQ05u8cTQClHE1Vehw

E-mail da secretaria executiva do I CoPU: encontroppgsurutai2020@gmail.com

\section{Edição dos anais}

Anderson Rodrigo da Silva

Loiany Gonçalves Costa

Arte e capa

Caio César de Oliveira Pereira 


\title{
Comissão Organizadora
}

\author{
Portaria n. 196, de 17 de setembro de 2020
}

Ricardo Diógenes Dias Silveira - Coordenador do PPG-ENEB e presidente da comissão Anderson Rodrigo da Silva - Diretor de Pós-Graduação, Pesquisa e Inovação e vice-presidente Marco Antônio Moreira de Freitas - Coordenador do PPGPP

Daniel de Paiva Silva - Coordenador do PPG-CRENAC

André Cirilo de Sousa Almeida - Docente do PPGPP

André Luis da Silva Castro - Docente do PPG-ENEB/CRENAC

Débora Astoni Moreira - Docente do PPG-ENEB/CRENAC

Cristiane Maria Ribeiro - Docente do PPG_ENEB/PROFEPT

Andressa Carneiro Nascimento - Discente do PPG-CRENAC

Caio César de Oliveira Pereira - Discente do PPG-CRENAC

Fernanda Paolla da Silva - Discente do PPG-CRENAC

Hamane Rodrigues da Silva Borges Freitas - PPG-ENEB

Loiany Gonçalves Costa - Discente do PPG-CRENAC

Tatiane de Andrade Resende - Discente do PPG-ENEB

Walter Baida Garcia Coutinho - Discente do PPGPP

\section{Comissão Científica}

André Cirilo de Almeida - Docente do PPGPP

André Luis da Silva Castro - Docente do PPG-CRENAC

Cristiane Maria Ribeiro - Docente do PPG-ENEB/PROFEPT

Daniel de Paiva Silva - Coordenador do PPG-CRENAC

Débora Astoni Moreira - Docente do PPG-ENEB/CRENAC

Érica Fernandes Leão Araújo - Docente do PPGPP

Ivandilson Pessoa Pinto de Menezes - Docente do PPG-PP/CRENAC

Tânia Maria de Moura - Docente do PPG-CRENAC

Marco Antonio Moreira de Freitas - Coordenador do PPGPP

Ricardo Diógenes Dias Silveira - Coordenador do PPG-ENEB

Marcos Fernandes Sobrinho - Docente do PPG-ENEB

Cleber Cezar de da Silva - Docente do PPG-ENEB 


\section{Programação}

com links para as transmissões de cada atividade

23 de outubro de 2020 - Fim das submissões de trabalhos

30 de outubro de 2020 - Prazo final para emissão de parecer pela Comissão Científica

\section{4 de novembro de 2020}

- Encerramento de inscrições

- 14:00, Ciência e Formação de Pesquisadores no Brasil

Prof. Dr. José Alexandre Felizola Diniz-Filho - Universidade Federal de Goiás

Mediador: Prof. Dr. Daniel de Paiva Silva - IF Goiano/Campus Urutaí

- 18:45, Abertura do evento

Prof. Dr. Paulo César Ribeiro da Cunha - Diretor-geral do Campus Urutaí;

Prof. Dr. Alan Carlos da Costa - Pró-Reitor de Pós-Graduação, Pesquisa e Inovação do IF Goiano;

Prof. Dr. Elias Monteiro de Padua - Reitor do IF Goiano.

Prof. Dr. Ricardo Diógenes Dias Silveira - IF Goiano/Campus Urutaí

Prof. Dr. Marco Antônio Moreira de Freitas - IF Goiano/Campus Urutaí

Prof. Dr. Daniel de Paiva Silva - IF Goiano/Campus Urutaí

- 19:00, Indicadores de qualidade da Pós-Graduação Profissional

Profa. Dra. Liliana Pena Naval - Universidade Federal do Tocantins

Dr. Geraldo José da Silva Junior - Fundo de Defesa da Citricultura (Fundecitrus)

Mediador: Prof. Dr. André Luis da Silva Castro - IF Goiano/Campus Urutaí

\section{5 de novembro de 2020}

- 08:00, Mesa-Redonda - Temas contemporâneos na Formação de Docentes Prof. Dr. Benjamin Xavier de Paula - Universidade de Federal de Uberlândia Profa. Dra. Maria Francisca da Cunha - Universidade Estadual de Goiás Mediadora: Profa. Dra. Cristiane Maria Ribeiro - IF Goiano/Campus Urutaí

- 13:30-16:00, Apresentações de trabalhos (pelos canais dos PPGs no YouTube)

- 17:30, Novas Abordagens para o Manejo da Resistência de Insetos à Táticas de Controle Prof. Dr. José Bruno Malaquias - Instituto de Biociências, UNESP-Botucatu Mediador: Prof. Dr. Marco Antônio Moreira de Freitas - IF Goiano/Campus Urutaí

- 18:30, Entomologia Digital: Inovação e Tecnologia no Manejo de Culturas Agrícolas Prof. Dr. Tavvs Micael Alves - Polo de Inovação do IF Goiano Mediador: Prof. Dr. Marco Antônio Moreira de Freitas - IF Goiano/Campus Urutaí

- 19:00, Desenvolvimento de produtos educacionais e avaliação da CAPES Profa. Dra. Ivanize Maria Rizzatti - Universidade Estadual de Roraima Mediador: Prof. Dr. Ricardo Diógenes Dias Silveira - IF Goiano/Campus Urutaí

- 20:30, Encerramento Mediador: Prof. Dr. Anderson Rodrigo da Silva - IF Goiano/Campus Urutaí 


\section{Índice}

Aspectos do conhecimento cultural da fitoterapia no Cerrado ..................................................................... 48

O uso do Kahoot! para o ensino remoto de razão e proporção em uma turma do $9^{\circ}$ ano ............................ 49

Distribuição geográfica da diversidade entomológica em matrizes ambientais .......................................... 50

Danos cromossômicos causados por concentrações ambientais relevantes de "mix" de poluentes em

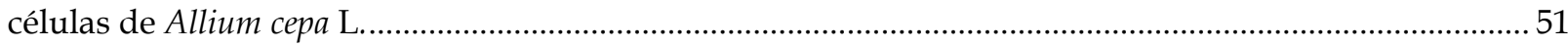

O uso do Catálogo de PEEFEM como material referencial ..................................................................... 52

Educação Precoce para crianças com deficiência auditiva/surdez .............................................................. 53

Aves Endêmicas do Cerrado em Unidades de Conservação no Brasil ......................................................... 54

Predição Espacial e Análise Temporal da Capacidade de Suporte de Carga dos Solos da Bacia do Rio

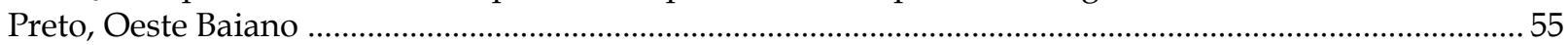

Aplicação de métodos para amostragem de mamíferos de médio e grande porte ........................................56

Aves de interesse conservacionista de um reservatório na região do médio rio Tocantins, Brasil............ 57

Diversidade da avifauna em áreas de influência da Usina Hidrelétrica de Estreito, Brasil .........................58

Efeitos dos nanoplásticos de poliestireno sobre Ctenopharyngodon idella (carpa capim) após exposição

individual e combinada com nanopartículas de óxido de zinco .......................................................................5 


\section{Índice de autores}

Abner Marcelino Silva __ 59

Abraão Tiago Batista Guimarães _ 59

Amanda Pereira da Costa Araújo _

Ana Flavia de Jesus Pinto___ 51

Anderson Rodrigo da Silva _ 55

André Bonadias Gadelha _ 52

Andressa Carneiro do Nascimento__ 48

Arthur A. Bispo 57,58

Caio César de Oliveira Pereira __ 48

Cláudia Amorim Madoz _ـ 53

Daniel de Paiva Silva $\longrightarrow 57,58$

Dione Carlos da Silva

Elias luiz Neves

Elto Aparecido Moreira

Fernanda Neves Estrela _ 59

Géssica Kalline Rodrigues Silva __ 54

Guilherme Malafaia __ 59

Ítalo Rômulo Mendes de Souza__ 55

Ivandilson Pessoa Pinto de Menezes __ 51

João Pedro Justino de Oliveira _— 56

Júlio Cesar Ferreira __ 49

Karla Dayane de L. Pereira _ 54, 57, 58

Letícia de Maria Oliveira Mendes__ 51

Lorrana Lucas Gomes Sampaio___ 51

Maike Oliveira Baía__ 51

Nayara Lima Batista

Peterson Trindade dos Santos _ 52,53

Rubens Pereira de Jesus _ـ 49

Saulo Santos Domingos 550,56

Tatiane de Andrade Resende__49, 52

Tenilce Gabriela da Silva __ 56

Tenilce Gabriela da Silva Alvarez _ 50

Thales Quintao Chagas_ 50,56

Thiarlen Marinho da Luz

Wallace Alves Cunha _ 48 


\title{
Aspectos do conhecimento cultural da fitoterapia no Cerrado
}

\author{
Andressa Carneiro do Nascimento ${ }^{1 *}$, Caio César de Oliveira Pereira ${ }^{1}$, Wallace Alves \\ Cunha ${ }^{1}$ \\ ${ }^{1}$ Departamento de Ciências Ambientais, Instituto Federal Goiano, Rodovia Geraldo Silva Nascimento, km 2.5, Zona Rural, \\ Caixa Postal 75790-000, Urutaí, Goiás, Brazil. \\ *Autor correspondente. E-mail: andressa-nha@hotmail.com
}

Desde o início da era antropocênica a humanidade retira do meio ambiente os recursos necessários para sobreviver. O conhecimento da fitoterapia, foi passado ao longo de gerações, através de um processo de ensino-aprendizagem natural. Todo este conhecimento empírico juntamente com mitos e rituais, formam parte importante das culturas locais. Grande parte da flora presente no bioma Cerrado apresenta potencial medicinal. Para o estudo do uso das espécies vegetais deve se considerar os contextos social e cultural como fator indispensável. O objetivo do trabalho é abordar o uso da fitoterapia no Cerrado, contextualizando a divulgação do conhecimento e, também, evidenciar o uso fitoterápico das espécies nativas no contexto atual da sociedade. A abordagem metodológica utilizada neste trabalho caracteriza-se como uma pesquisa sistemática qualitativa, de cunho exploratório. Foram coletados dados da literatura. Selecionamos artigos do começo da década de 1990 aos tempos atuais que abordam as plantas medicinais como manifestação cultural no Cerrado e informações técnico científica. Buscamos relacionar as manifestações culturais que ocorrem no Cerrado com a enorme diversidade biológica pertencente a este hotspot de biodiversidade e formas de divulgação. O Cerrado tem sofrido modificações significativas nas últimas quatro décadas, influenciadas pelo desenvolvimento do seu território. Tais modificações alteram a dinâmica cultural em diversos ambientes, podendo causar a extinção dos aspectos culturais tradicionais, e também de espécies vegetais, mesmo antes de serem conhecidas taxonomicamente e avaliadas pela ciência. Apesar da evolução da medicina moderna e acesso a fármacos sintéticos, a população mantém o interesse pelo uso do "natural". Trabalhos vêm sendo desenvolvidos em diversas regiões do Brasil, para chamar a atenção para o uso popular de plantas medicinais. Atualmente observa-se o surgimento de ferramentas e meios de buscar o conhecimento fitoterápico. O acesso à internet é um avanço tecnológico extremamente importante nesse aspecto. Entretanto, existem muitas informações errôneas ou obsoletas de fácil acesso, sendo assim, evidencia-se que ainda há a necessidade de pesquisar e compartilhar informações corretas, de preferência que tenham sido comprovadas pela ciência. Destaca-se, também, a necessidade de maior incentivo nas pesquisas com plantas medicinais pelo governo, sendo que, das mais de 300.000 espécies de plantas existem no mundo, é estimado que aproximadamente $15 \%$ foram avaliadas para determinar seu potencial farmacológico.

Palavras-chave: Plantas medicinais; Divulgação científica; Biodiversidade; Bioma Cerrado.

\begin{abstract}
Aspects of cultural knowledge of phytotherapy in the Cerrado
Considerable part of flora present in the Brazilian Savanna has medicinal potential. The empirical knowledge of phytotherapy, together with myths and rituals, form an important part of local cultures. The Cerrado (Brazilian Savanna) has undergone modifications that change cultural dynamics in different environments, and can cause the extinction of cultural aspects, and likewise of plant species, even before they were known taxonomically and evaluated by science. With the increasing use of technology, ways of seeking phytotherapeutic knowledge are emerging. However, erroneous information is easily accessible, so it is clear that there is still a need to research and share scientifically proven information.
\end{abstract}

Key words: Medicinal plants, Scientific Communication, Biodiversity, Brazilian Savanna.

Agradecimentos: À prof. Dr. Ana Paula Silva Siqueira. Ao prof. Dr. Ivandilson Pessoa Pinto de Menezes. Ao

Programa de Pós graduação em Conservação de Recursos Naturais do Cerrado. Ao Instituto Federal Goiano.

Para citar:

Nascimento, A. C., Pereira, C. C. O., \& Cunha, W. A. (2020). Aspectos do conhecimento cultural da fitoterapia no Cerrado. In: Anais do I Colóquio da Pós-Graduação do IF Goiano - Campus Urutaí, 4 a 5 de novembro de 2020

(p. 48). Urutaí - GO: Multi-Science Journal, 3(3). DOI: https:// doi.org/10.33837/msj.v3i3.1349 


\title{
O uso do Kahoot! para o ensino remoto de razão e proporção em uma turma do $9^{\circ}$ ano
}

\author{
Tatiane de Andrade Resende ${ }^{1 *}$, Rubens Pereira de Jesus², Júlio Cesar Ferreira ${ }^{3}$ \\ ${ }^{1}$ Mestranda do Programa de Pós-Graduação em Ensino para Educação Básica, Instituto Federal Goiano, Campus Urutaí \\ ${ }^{2}$ Mestrando do Programa de Pós-Graduação em Ensino para Educação Básica, Instituto Federal Goiano, Campus Urutaí \\ ${ }^{3}$ Docente do Programa de Pós-Graduação em Ensino para Educação Básica, Instituto Federal Goiano, Campus Urutaí \\ *autora apresentadora: tateresende@hotmail.com
}

\begin{abstract}
O uso das Tecnologias Digitais de Informação e Comunicação (TDICs) nas situações de ensino tem despertado a atenção de muitos profissionais da educação, principalmente diante da constatação do desinteresse dos alunos quando empregado metodologias tradicionais de ensino. Esta situação é agravada no cenário de ensino remoto provocado pela crise sanitária levando o professor a se reinventar e utilizar metodologias ativas que buscam a aprendizagem significativa à luz de David Ausubel. Uma das ferramentas de TDICs com promissora capacidade de utilização como apoio ao ensino é a kahoot! Plataforma de aprendizagem baseada em jogos de perguntas e respostas com pontuação, interação e ranqueamento. Este trabalho trata-se de uma pesquisa qualitativa em uma turma com 33 alunos do nono ano do Colégio Estadual da Polícia Militar de Goiás (CEPMG) em Morrinhos. Objetivou-se verificar a participação e engajamento dos alunos utilizando o Kahoot como ferramenta para integrar as TDICs nas situações de ensino. Para o desenvolvimento da intervenção pedagógica, foi preparado um quiz online composto por 20 questões de múltipla escolha, com 1 alternativa correta e enfoque em razão e proporção, denominado BÔNUSQUIZ. Foi definido o tempo máximo de 60 segundos para responder cada questão. As questões foram elaboradas de acordo com as atividades postadas diariamente na plataforma GR8. Para a aplicação do quiz foram necessárias duas aulas de matemática: na primeira aula foi esclarecido a funcionalidade do aplicativo, as regras e também disponibilizado o código de acesso para os alunos e na segunda aula foi aplicado o Kahoot!. Ao término do quiz disponibilizou-se um placar com os resultados na ordem crescente da pontuação. Foi registrado o percentual total de participantes ativos na atividade. Com as observações das participações dos alunos, verificou-se que 22 alunos $(66,7 \%$ dos alunos matriculados) participaram ativamente do quiz e das aulas remotas. Todos os alunos que estavam online concluíram a tarefa em tempo hábil. Os resultados foram sastifatórios e concluiu-se o uso das TDICs nas aulas tornam as participações mais ativas, pois os alunos sentem-se atraídos e estimulados. Dessa forma, observa-se fortes indícios de que plataforma Kahoot! permite abordar todo o conteúdo da disciplina de modo mais dinâmico e atrativo, ainda que remoto, resultando um bom estímulo para uma aprendizagem significativa.
\end{abstract}

Palavras-chave: Kahoot!, Ensino da Matemática, Aprendizagem significativa.

The use of Kahoot! For the remote teaching of reason and proportion in one class of Ninth grade. This work was developed using Kahoot! In one class with 33 students of ninth grade of the afternoon shift at Goiás State Military Police College (CEPMG) in Morrinhos. For the application of the quiz, two math classes were needed: In the fisrt lesson the funciotionality of the application was clarified, the rules and access code was also made available to students, and in the other class, the Kahoot! was applied. The results were satisfactory and it is conclude that the use of TDICs in classes makes the participations more active, because the students are attracted and stimulated.

Keywords: Kahoot!, Math teaching, Meaningful learning.

Para citar:

RESENDE, Tatiane de Andrade \& JESUS, Rubens Pereira de \& FERREIRA, Júlio César. (2020). O uso do Kahoot! para o ensino remoto de Matemática em uma turma do $9^{\circ}$ ano. In: Anais do I Colóquio da PósGraduação do IF Goiano - Campus Urutaí, 4 a 5 de novembro de 2020 (p. 49). Urutaí - GO: Multi-Science Journal, 3(3). DOI: https://doi.org/10.33837/msj.v3i3.1349 


\title{
Distribuição geográfica da diversidade entomológica em matrizes ambientais
}

\author{
Saulo Santos Domingos 1*,Thales Quintao Chagas", Tenilce Gabriela da Silva Alvarez', \\ Nayara Lima Batista ${ }^{1}$, Elto Aparecido Moreira ${ }^{1}$, \\ ${ }^{1}$ Programa de Pós-Graduação de Conservação de Recursos Naturais do Cerrado, Instituto Federal Goiano Campos Urutaí, \\ Urutaí, Goiás, Brasil \\ ${ }^{*}$ Autor correspondente. E-mail: sandownin@hotmail.com
}

\begin{abstract}
A degradação ambiental tem levado a formação de fragmentos florestais com presença de matrizes com área florestal preservada, transição e de pastagem. A entomofauna é indicador de alteração ambiental, uma vez que essas espécies são influenciadas pelas mudanças de hábitats. Este estudo tem como objetivo realizar um levantamento na distribuição de indivíduos de insetos em escala de ordens em três matrizes ambientais (mata, pastagem e ambiente de transição), partindo da hipótese que o ambiente com maior cobertura vegetal (ambiente preservado) possui maior riqueza e abundância relativa das ordens de insetos. O trabalho foi conduzido no município de Urutaí, Goiás, nas imediações do Instituto Federal Goiano no setor de bovinocultura; foram instalados 45 pitfall (armadilhas de queda) com água e detergente distribuídos nos três ambientes, sendo 5 pitfall com iscas (banana) e 10 sem iscas em um período de um dia. Para cada armadilha foram registrados os dados referentes as coordenadas geográficas "UTM" durante o experimento de campo. As identificações dos insetos ocorreram no laboratório de entomologia do Instituto Federal Goiano - Campus Urutaí, GO, chegando a nível de Ordem. As análises estatísticas foram realizadas para comparar o número de indivíduos das ordens mais frequentes em cada ambiente através do teste $\mathrm{t}$ - student, com posterior análise da abundância por área e a distribuição especial, por meio do software livre R. Identificou a presença de nove ordens de invertebrados da Classe Insecta, sendo elas: Blattodea, Coleoptera, Dermaptera, Diptera, Hemiptera, Homoptera, Hymenoptera, Isoptera e Orthoptera. Os indivíduos que predominaram em todos os ambientes foram das ordens Hymenoptera, Coleoptera e Orthoptera. Dos 45 pitfalls instalados, 44 registraram a presença de representantes da ordem Hymenoptera, pertencentes à família Formicidae (formigas). A abundância desses indivíduos (Hymenoptera) nos ambientes de pastagem e mata $(t=3.0642, \mathrm{df}=15.381$, $\mathrm{p}$-value $=0.00769)$ apresentou diferença significativa. Outra ordem que se destacou nas coletas trata-se da ordem Coleoptera, que foi registrada em 9 pitfalls e a ordem Orthoptera foi registrada em 7 pitfalls dos 45 instalados, além delas não apresentarem diferença estatística em nenhum dos ambientes. Assim concluimos que ambiente de intermediário (transição) foi o ambiente com maior abundância e riqueza de indivíduos nas áreas estudadas, a hipótese inicial do estudo foi negada, uma vez que o ambiente com maior abundância e riqueza não foi o ambiente mais preservado.
\end{abstract}

Palavras-chave: degradação ambiental; fragmentos florestais; entomofauna.

Geographic distribution of entomological diversity in environmental matrices Environmental degradation has led to the formation of forest fragments with the presence of matrices with preserved forest area, transition and pasture. The work was carried out in the municipality of Urutaí, Goiás, in the vicinity of the Federal Goiano in Institute the bovine culture sector; 45 pitfalls (drop traps) were installed with water and detergent distributed in the three environments, 5 pitfalls with baits (banana) and 10 without baits in a period of one day.the initial hypothesis of the study was denied, since the environment with the greatest abundance and wealth was not the most preserved environment.

Keywords: Ambiental degradation; forest fragments; entomofauna

\footnotetext{
Agradecimentos: Agradeço ao Prof Dr. Anderson Silva pela orientação do trabalho

Para citar:

Domingos et al. (2020). Distribuição geográfica da diversidade entomológica em matrizes ambientais. In: Anais do I Colóquio da Pós-Graduação do IF Goiano - Campus Urutaí, 4 a 5 de novembro de 2020 (p. 50). Urutaí GO: Multi-Science Journal, 3(3). DOI: https:// doi.org/10.33837/msj.v3i3.1349
} 


\title{
Danos cromossômicos causados por concentrações ambientais relevantes de "mix" de poluentes em células de Allium cepa L.
}

\author{
Maike Oliveira Baía ${ }^{1 *}$, Lorrana Lucas Gomes Sampaio ${ }^{1}$, Letícia de Maria Oliveira \\ Mendes${ }^{1}$, Elias luiz Neves ${ }^{1}$, Ana Flavia de Jesus Pinto ${ }^{1}$, Ivandilson Pessoa Pinto de \\ Menezes ${ }^{1}$. \\ Instituto Federal Goiano - Campus Urutaí ${ }^{1}$ \\ ${ }^{*}$ Autor correspondente. E-mail: maikegfc20@gmail.com
}

\begin{abstract}
No decorrer dos últimos anos é possível observar a pressão para produção de alimentos, serviços e produtos industrializados. A demanda por produção vem sendo acompanhada pelos avanços na expansão das fronteiras agrícolas e na geração de produtos de consumo, levando o aumento do uso extensivo de insumos agrícolas e na produção de resíduos industriais, respectivamente, os quais são descartados ou chegam no meio ambiente onde inevitavelmente não são encontrados individualmente, mas sim misturados. Objetivamos testar se a exposição de água contendo mix de poluentes em concentrações ambientais relevantes pode causar fragmentação nuclear e o aumento de anomalias mitóticas. Para isso utilizamos o modelo de planta Allium cepa, amplamente utilizados em estudos eco(toxicológicos). Setenta e cinco bulbos foram distribuídos e expostos a cinco distintos tratamentos durante 24, 48 e 72 h com n= 5: G1- Controle Negativo apenas com água potável; G2 - Controle Positivo com reagente citotóxico (ciclofosfamida, $10 \mathrm{mg} / \mathrm{mL}$ ); G3 - com uma mistura de 15 contaminantes em concentrações ambientais relevantes; G4 - uma diluição de G3 na ordem de 1/2; e G5 - uma diluição de G3 na ordem de 1/4. A toxicologia será avaliada através do efeito do mix de poluente usando o índice mitótico (IM) e de anormalidade cromossômica (IAC) estimados pela contagem de 15 mil células por tratamento. As lâminas foram preparadas usando o método de esmagamento, coradas com Saffranin a $45 \%$ e examinadas em microscopia óptica. Até o presente momento foi observado em todas as diferentes concentrações de mix de poluente e controle positivo diferentes anormalidades cromossômicas em todas as fases da divisão celular (quebra cromossômica, ponte cromossômica, perturbação do fuso, aderência cromossômica, cromossomos retardatários, além de micronúcleos). Não sendo observado anormalidades no controle negativo. Com esses resultados preliminares, observamos que o mix de poluentes mesmo em baixa concentração e curto período de tempo tem aumentado o número de anormalidade do DNA em células meristemáticas de Allium cepa, indicando seu potencial toxicológico.
\end{abstract}

Palavras-chave: Impacto ambiental; Cromossomos; Allium cepa.

Chromosomal damage caused by relevant environmental concentrations of pollutants in Allium cepa $L$ cells.

It will be evaluated whether the exposure of water containing a mix of pollutants in relevant environments can cause nuclear fragmentation and the increase of mitotic anomalies. The cytotoxic and genotoxic effect will be evaluated in meristematic cells of $A$. cepa root exposed to five treatment groups during 24, 48 and $72 \mathrm{~h}$ with $\mathrm{n}=5$ : Negative Control (drinking water); Positive Control (cyclophosphamide); G3 a mixture of 15 contaminants at relevant environmental concentrations; G4 - a dilution of G3 in the order of 1/2; and G5 - a dilution of G3 in the order of $1 / 4$.

Key words: Environmental impact; Chromosome; Allium cepa.

Agradecimentos: Este trabalho foi realizado com o auxílio do Instituto Federal Goiano - Campus Urutaí.

Para citar:

Baía, M. O., Sampaio, L. L. G., Mendes, L. M. O., Neves, E. L., Pinto, A. F. J., Menezes, I. P. P. (2020). Danos cromossômicos causados por concentrações ambientais relevantes de "mix" de poluentes em células de Allium cepa L. In: Anais do I Colóquio da Pós-Graduação do IF Goiano - Campus Urutaí, 4 a 5 de novembro de 2020 (p. 51). Urutaí - GO: Multi-Science Journal, 3(3). DOI: https://doi.org/10.33837/msj.v3i3.1349 


\title{
O uso do Catálogo de PEEFEM como material referencial
}

\author{
Tatiane de Andrade Resende ${ }^{1 *}$, Peterson Trindade dos Santos ${ }^{2}$, Dione Carlos da Silva ${ }^{3}$, \\ André Bonadias Gadelha ${ }^{4}$ \\ ${ }^{1}$ Mestranda do Programa de Pós-Graduação em Ensino para Educação Básica, Instituto Federal Goiano, Campus Urutaí \\ ${ }^{2}$ Mestrando do Programa de Pós-Graduação em Ensino para Educação Básica, Instituto Federal Goiano, Campus Urutaí \\ ${ }^{3}$ Mestrando do Programa de Pós-Graduação em Ensino para Educação Básica, Instituto Federal Goiano, Campus Urutaí \\ ${ }^{4}$ Docente do Programa de Pós-Graduação em Ensino para Educação Básica, Instituto Federal Goiano, Campus Urutaí \\ *Autora apresentadora: tateresende@hotmail.com
}

O ensino de Física no Ensino Médio (EM) é um desafio tanto para os professores como para os estudantes, principalmente, no que tange às estratégias de intervenção metodológica para a efetivação de uma abordagem significativa. O presente estudo encontra-se em desenvolvimento e tem como objetivo criar um material de suporte com estratégias metodológicas para o ensino de Física no EM. Em especial, nas aulas de Física, observa-se que o processo de ensino-aprendizagem ainda está centralizado na figura do professor, o que pode contribuir para a redução da participação ou até mesmo desinteresse dos estudantes. O currículo convencional está orientado para palestras, com ênfase no aprendizado passivo, contrapondo o aprendizado ativo onde as abordagens pedagógicas são centradas no aluno, dando foco nele o no que ele faz. Contudo, o aprendizado ativo precede um ambiente de aprendizado estruturado, no qual ele, enquanto docente, além de figurar como aprendiz neste processo, também permite que o discente assuma a posição de protagonista do mesmo. Neste cenário, o uso de estratégias docentes a partir de autoexplicações tem se mostrado eficiente na melhora o aprendizado, sendo importante que os estudantes tenham a oportunidade de ouvir (e ler) e falar (ou escrever) durante as aulas. No exercício do fazer pedagógico é intensa a busca, por parte do professor, por métodos, técnicas e tecnologias que o auxiliem na elaboração de suas aulas, contribuindo para que se apresentem como recurso motivador no processo de ensino-aprendizagem dos estudantes. Os Produtos Educacionais para o Ensino de Física no Ensino Médio (PEEFEM) será desenvolvido em formato digital (E-Book), apresentado com textos explicativos e imagens de suporte. O catálogo PEEFEM contará com 12 propostas de produtos educacionais relacionados ao ensino da Física no EM apresentados em Mestrados Profissionais em Educação e em Ensino no período de 2010 a 2020, listados e organizados para subsidiar o planejamento de aula e os projetos dos professores de Física. Sendo assim, o PEEFEM apresentará como uma fonte rica de consulta, sendo uma boa oportunidade de trazer os resultados das pesquisas na área em sala de aula tradicional ou no espaço dos laboratórios. Ressalta-se que, o uso da tecnologia é importante para o êxito do processo de ensino-aprendizagem e por meio dela os professores conseguem aproximar o que está sendo ensinado à realidade prática dos alunos. Neste sentido, isso pode suprir a lacuna existente no que diz respeito ao acesso de materiais que permitem inovação.

Palavras-chave: Produto Educacional, Catálogo, Física, Ensino Médio.

The use of the PEEFEM Catalog as reference material

The present study is ongoing and aims to create a support catalog with methodological strategies for teaching physics in High School. Educational Products for Teaching Physics in High School (EPTPHS) will be digitally developed (E-Book), presenting explanatory texts illustrated by supporting pictures. The EPTPHS catalog will feature 12 approaches for educational products related to the teaching of Physics in high school, being these products available from Professional Masters Degree (Education and/or Teaching Programs) from 2010 to 2020, listed and organized to support the planning schedule and projects of physics teachers.

Key-words: Educacional Products, Catalog, Physics, High School.

Para citar:

RESENDE, Tatiane de Andrade \& SANTOS, Peterson Trindade dos \& SILVA, Dione Carlos da \& GADELHA, André Bonadias. (2020). O uso do catálogo de PEEFEM como material referencial. In: Anais do I Colóquio da Pós-Graduação do IF Goiano - Campus Urutaí, 4 a 5 de novembro de 2020 (p. 52). Urutaí - GO: Multi-Science Journal, 3(3). DOI: https://doi.org/10.33837/msj.v3i3.1349 


\title{
Educação precoce para crianças com deficiência auditiva/surdez
}

\author{
Cláudia Amorim Madoz ${ }^{*}$, Peterson Trindade dos Santos² \\ ${ }^{1}$ Pedagoga. Especialista em Educação Especial e Inclusiva. Professora efetiva da SEEDF. \\ ${ }^{2}$ Mestrando do Programa de Pós-Graduação em Ensino para Educação Básica, Instituto Federal Goiano, Campus Urutaí \\ *autora apresentadora: claudiamadoz25@gmail.com
}

\begin{abstract}
A Educação Precoce (EP) é um programa educacional ofertado pela Secretaria de Estado de Educação do Distrito Federal (SEEDF) voltado a proporcionar à criança experiências significativas, a partir do seu nascimento, que promovam o desenvolvimento máximo do seu potencial. Diante disso, esse trabalho investiga a importância da EP para o desenvolvimento linguístico de crianças surdas ou deficientes auditivas, na faixa etária de zero a três anos e onze meses. Esta pesquisa partiu da seguinte problemática: Como tem sido trabalhada a criança com surdez ou deficiência auditiva na EP para a promoção da sua inclusão na Educação Infantil? A hipótese levantada neste estudo é de que a educação precoce ainda está voltada para outras deficiências e não para a educação linguística da criança surda. O objetivo geral é compreender como é realizado o processo de Educação Precoce na prática pedagógica dos professores de crianças com surdez ou deficiência auditiva, na idade de 0 a 3 anos e 11 meses, em duas escolas que oferecem o programa: Centro de Ensino Especial 2 de Brasília (CEE 2 de Brasília) e Escola Bilíngue de Taguatinga (EBT), unidades escolares da SEEDF. Tomamos como referencial teórico a Psicologia Histórico-Cultural que vê a criança com deficiência com as mesmas possibilidades de desenvolvimento como qualquer outra, mas que este desenvolvimento se dará de forma diferenciada, levando em consideração suas especificidades, para tanto precisa do professor agente mediador desenvolvimento e da aprendizagem. Trata-se de um estudo exploratório-descritivo, de abordagem qualitativa. Iniciamos a análise documental pelo estudo dos principais documentos orientadores e normativos da política da Educação Precoce no DF, estabelecendo um diálogo com as políticas públicas de educação bilíngue para surdos. Para além, analisaremos os resultados e dados da prática pedagógica dos professores, tomando como referência as categorias: conceitos, metodologia, recursos e obstáculos. Os dados serão coletados por observação de crianças surdas ou com deficiência auditiva em atendimento na sala de aula, entrevistas semiestruturadas e questionários com questões abertas. As entrevistas serão feitas com os gestores, coordenadores e profissionais da equipe especializada de apoio à aprendizagem e os questionários aplicados aos professores. Diante disso, a pretensão é constatar a necessidade de um maior investimento na formação dos professores da educação precoce, principalmente no que se refere ao trabalho com a criança com surdez e deficiência auditiva. É preciso que institua uma política de formação aos professores que promova conhecimentos teóricos e práticos para favorecer a aprendizagem precoce da Língua Brasileira de Sinais-LIBRAS às crianças com surdez ou deficiência auditiva.
\end{abstract}

Palavras-chave: Educação Precoce; Deficiência Auditiva/Surdez; Libras; Inclusão.

Early education for children with hearing loss / deafness

Early Education, an educational program, aimed at providing children with meaningful experiences that promote their maximum development. This research started from the problem: How has the child with deafness / hearing impairment been worked in PE for their inclusion in Early Childhood Education? The general objective is to understand how the PE process is carried out in the pedagogical practice of teachers of children with deafness / hearing impairment in two schools: Centro de Ensino Especial 2 de Brasília and Escola Bilingual de Taguatinga. The documentary analysis started from the study of the guiding and normative documents of the EP establishing a dialogue with the public policies of bilingual education.

Key-words: Early Education; Hearing Impairment / Deafness; Libras; Inclusion.

Para citar:

MADOZ, C. A. \& SANTOS, P. T. (2020). Educação Precoce para crianças com deficiência auditiva/surdez. In: Anais do I Colóquio da Pós-Graduação do IF Goiano - Campus Urutaí, 4 a 5 de novembro de 2020 (p. 53). Urutaí GO: Multi-Science Journal, 3(3). DOI: https:// doi.org/10.33837/msj.v3i3.1349 


\title{
Aves Endêmicas do Cerrado em Unidades de Conservação no Brasil
}

\author{
Géssica Kalline Rodrigues Silva ${ }^{1}$, Karla Dayane de L. Pereira ${ }^{2}$ \\ 1 Programa de Pós-Graduação em Conservação de Recursos Naturais do Cerrado (PPG-CRENAC), IF Goiano, Rod. Geraldo \\ Silva Nascimento KM 2,5 Zona Rural, Urutaí, GO, 75790-000, Brasil. \\ ${ }^{2}$ Programa de Pós-Graduação em Conservação de Recursos Naturais do Cerrado (PPG-CRENAC), IF Goiano, Rod. Geraldo \\ Silva Nascimento KM 2,5 Zona Rural, Urutaí, GO, 75790-000, Brasil. ORCiD: https://orcid.org/0000-0003-1578-89480 \\ **Autor correspondente. E-mail: gessicakalline_18@hotmail.com
}

As Unidades de Conservação (UCs) constituem instrumentos para a conservação da biodiversidade, em especial, de espécies endêmicas e de importância conservacionista. No Brasil, existem 2081 UCs de instância Federal e Estadual, onde apenas 173 estão em domínio do Bioma Cerrado. Esse estudo, apresenta uma revisão da presença atual das aves endêmicas do Cerrado em UCs federais e estaduais por todo o Brasil. Apresentamos também, o número das UCs pesquisadas, com destaque de informações àquelas que possuem registros de uma ou mais espécies focais deste estudo. A revisão foi realizada em 2020. Foram consideradas as 30 espécies de aves endêmicas do Cerrado, listadas de 1995-2002. Os registros de ocorrência em UCs foram obtidos a partir de literaturas, planos de manejo das UCs, Listas Vermelhas de Espécies Ameaçadas (IUCN e ICMBio), Sistema Nacional de Unidades de Conservação, bancos de dados Online, além de observações pessoais de campo. Registros com mais de duas décadas foram desconsiderados. Para amostragem de presença e/ou ausência, cada espécie foi contabilizada uma única vez por UCs. Foram pesquisadas 183 UCs federais e estaduais no Brasil. Destas, 153 estão em áreas de Cerrado, onde 134 são de domínio do bioma. Do total de UCs pesquisadas $(\mathrm{n}=183)$, em apenas 40,4\% $(n=74)$ foram encontrados registros recentes de aves endêmicas do Cerrado. Das UCs com presença de aves endêmicas, 75,7\% estão em domínio do Cerrado, seguidos de 24,3\% em domínio de biomas adjacentes. O maior número de registros foi observado em UCs estaduais ( $\mathrm{n}=$ 47). As UCs de proteção integral foram as mais representativas ( $n=42$ registros), enquanto as de uso sustentável somaram 32 registros. Os parques (15 federais e 21 estaduais) foram a categoria com maior número de registros de aves endêmicas. Todas as 30 espécies estão presentes em UCs. As espécies registradas em maior número de UCs foram: Alipiopsitta xanthops (Spix, 1824) $(\mathrm{n}=16)$, Charitospiza eucosma (Oberholser, 1905) ( $\mathrm{n}=14)$ e Antilophia galeata (Lichtenstein, 1823) ( $\mathrm{n}=13)$. Cada uma das espécies, Hydropsalis candicans (Pelzeln, 1867) e Synallaxis simoni (Hellmayr, 1907), foram contabilizadas em apenas uma área protegida (parques estadual e federal). Algumas aves endêmicas do Cerrado são raras e/ou incomuns, estando praticamente restritas às áreas protegidas. No entanto, o Cerrado ainda é um bioma com baixo percentual de áreas protegidas. Dessa forma, não somente a criação de UCs, mas ações de fiscalização e manutenção destas, são indispensáveis à conservação da biodiversidade.

Palavras-chave: áreas protegidas; avifauna; distribuição; ocorrência.

Endemic birds of the Cerrado in Conservation Units in Brazil

Here, we review the distribution of the presence of endemic birds from the Cerrado in Conservation Units in Brazil. Records from the past two decades were considered. Altogether, recent records were found in only 74 Conservation Units distributed throughout Brazil. The largest number of records was observed in state conservation units, under the domain of the Cerrado Biome. All birds endemic to the Cerrado were found in Conservation Units.

Key words: protected areas; birdlife; distribution; occurrence.

Para citar:

Silva, G. K. R., \& Pereira, K. D. L. (2020). Aves endêmicas do Cerrado em Unidades de Conservação no Brasil. In: Anais do I Colóquio da Pós-Graduação do IF Goiano - Campus Urutaí, 4 a 5 de novembro de 2020 (p. 54). Urutaí - GO: Multi-Science Journal, 3(3). DOI: https:// doi.org/10.33837/msj.v3i3.1349 


\title{
Predição Espacial e Análise Temporal da Capacidade de Suporte de Carga dos Solos da Bacia do Rio Preto, Oeste Baiano
}

\author{
Ítalo Rômulo Mendes de Souza ${ }^{1^{*}}$, Anderson Rodrigo da Silva ${ }^{(i)}$ 2, \\ ${ }^{1}$ Mestre em Conservação de Recursos Naturais do Cerrado (PPG-CRENAC), Instituto Federal Goiano - Campus Urutaí, \\ Rod. Geraldo Nascimento, Km-2,5, Zona Rural, Urutaí - GO, 75.790-000, Brasil. \\ 2 Docente do PPG-CRENAC, Instituto Federal Goiano - Campus Urutaí, \\ *Autor correspondente. E-mail: ítalo_romulo@hotmail.com
}

Estudos sobre as alterações físicas/estruturais do solo e de comportamento compressivo são de grande importância no âmbito da conservação desse recurso natural. Nesse sentido, a pressão de pré-consolidação (op) é considerada como indicativo da capacidade de suporte de carga (CSC) que um solo pode tolerar em termos de pressões superficiais, em especial provenientes do tráfego agrícola. O objetivo deste trabalho foi realizar sensoriamento remoto espacial e avaliação temporal da capacidade de suporte de carga de solos da Bacia do Rio Preto, Oeste baiano, sob diferentes tipos de uso e cobertura. Utilizou-se técnicas de sensoriamento remoto com cenas do satélite LANDSAT 8 (OLI) para classificação de uso e cobertura do solo (classificação supervisionada) e modelos de predição da capacidade de suporte de carga disponíveis na literatura especializada para solos brasileiros, em função do teor de argila (SoilInfo, www.isric.org) e da umidade do solo na camada superficial $(\sim 5 \mathrm{~cm})$ fornecida pelo satélite Soil Moisture Active Passive - SMAP/NASA, com resolução espacial de $9 \mathrm{Km}$. Os dados de umidade foram obtidos mensalmente, num recorte temporal de 2016 a 2017. Obtidas as estimativas de CSC para cada classe de uso e cobertura, foram calculados intervalos de $95 \%$ de confiança para a média de cada classe, para comparações estatísticas. Verificou-se alta variação de CSC entre os meses, com maiores valores no período seco, indicando influência da sazonalidade da região. Em 2016, a capacidade de suporte de carga (CSC) variou e entre 169 e $258 \mathrm{kPa}$, sendo as tensões mais elevadas nos meses de fevereiro a julho, comportamento que pode estar atrelado com a redução de precipitação nestes meses com registros de 33,8 mm e 31,5 mm respectivamente. As culturas anuais/pecuária alcançaram média de 221,71 kPa em 2017, podendo ter relação com a utilização intensificada de maquinário agrícola, adoção de sistemas de manejo do solo e pisoteio de animais de grande porte. Conclui-se que, nos anos estudados maiores valores médios de tensões na classe de culturas anuais/pecuária com 226,57 kPa; Formação florestal / savânica com 220,24 kPa (referência) e 219,10 kPa para Formação campestre/pastagem. A avaliação da CSC por tipo de uso do solo pode evitar processos de deformação do física/estrutural e indicar recomendações entre as trações e pressões de inflação de maquinários, visando preconizar a intensidade e período do tráfego levando em consideração a concordância com as pressões superficiais do que se suporta o solo.

Palavras-chave: Capacidade de Suporte de Carga, Cerrado, SMAP, Tensão de Pré-Consolidação

Spatial Prediction and Temporal Analysis of Load Bearing Capacity of Soils in the River Black Basin, West Bahia

The pre-consolidation pressure is considered indicative of the load-bearing capacity (LBC) that a soil can tolerate in terms of surface pressures. With this work, remote sensing techniques and LBC prediction models available in the literature for Brazilian soils were applied in a west Bahia (Central Brazil) basin. We predicted the highest values of LBC during the dry season. In the years studied it is noted that the class annual crops / livestock showed higher CSC in the years studied $(226,48$ and $226.67 \mathrm{kPa})$ while the class Forest / countryside formation (reference) presented 222.61 and $217.87, \mathrm{kPa}$ respectively .

Key words: Load-bearing capacity; Cerrado; SMAP; Preconsolidation pressure.

Para citar:

Souza, I. R. M. \& Silva, A. R. (2020). Predição Espacial e Análise Temporal da Capacidade de Suporte de Carga dos Solos da Bacia do Rio Preto, Oeste Baiano. In: Anais do I Colóquio da Pós-Graduação do IF Goiano Campus Urutaí, 4 a 5 de novembro de 2020 (p. 55). Urutaí - GO: Multi-Science Journal, 3(3). DOI:

https://doi.org/10.33837/msj.v3i3.1349 


\title{
Aplicação de métodos para amostragem de mamíferos de médio e grande porte
}

\author{
Thales Quintao Chagas ${ }^{1 *}$, Tenilce Gabriela da Silva Alvarez ${ }^{1}$, Nayara Lima Batista ${ }^{1}$, Elto \\ Aparecido Moreira' ${ }^{1}$, Saulo Santos Domingos ${ }^{1}$, João Pedro Justino de Oliveira ${ }^{1}$ \\ ${ }^{1}$ Programa de Pós-Graduação de Conservação de Recursos Naturais do Cerrado, Instituto Federal Goiano Campos Urutaí, \\ Urutai, Goiás, Brasil \\ *Autor correspondente. E-mail: thalesquintao14@gmail.com
}

\begin{abstract}
O estudo corresponde a uma atividade desenvolvida durante a disciplina de métodos para inventário da biodiversidade ofertada pelo programa de pós-guraduação em Conservação de Recursos Naturais do Cerrado (PPGCRENAC). Na disciplina, foram discutidas metodologias de amostragem para os principais grupos faunísticos bioindicadores de qualidade ambiental (ornitofauna, mastofauna, entomofauna, ictiofauna e herpetofauna). Assim, o presente estudo teve por objetivo aplicar métodos para amostragem de mastofauna (médios e grandes), cumprindo assim a parte prática da disciplina. Ressalta-se que o intuito da presente atividade foi apenas aplicar os métodos discutidos na teoria e não inventariar a mastofauna local, pois para isso o esforço amostral deveria ser maior. Foram escolhidas três áreas de estudo identificadas como áreas A, B e C ambas com cerrado nativo e distintas fitofisionomias vegetais. Para amostrar a diversidade mastofaunistica das áreas foram feitos 2 transectos lineares $(1 \mathrm{~km})$ em cada uma delas, onde os animais foram identificados através de observações diretas (avistamento) e através de observações indiretas (pegadas, tocas e fezes). Cada ponto estudado contou com uma câmera trap por 10 dias (iscadas com atrativos como frutas e carnes). Além dos métodos citados, foram feitas entrevistas com moradores da região. Com um esforço amostral de 30h em campo e de $720 \mathrm{~h}$ de câmera trap (totalizando 750h) foi registrada uma riqueza de 18 espécies. Nove espécies foram registradas através dos transectos e cinco foram registradas pelas câmeras trap. Além destas, quatro espécies foram relatadas pelos moradores da região. A Ordem Cingulata apresentou a maior diversidade (5 spp.) seguida da Ordem Carnívora (4 spp.). Três das espécies registradas são ameaçadas de extinção, sendo elas Puma concolor, Myrmecophaga tridactyla e Priodontes maximus. A partir dos resultados obtidos, conclui-se que os métodos foram aplicados com eficiência e que a proposta da disciplina foi cumprida. Além de propiciar a vivência em campo e interação de profissionais de diferentes áreas de atuação, atividades práticas como essa podem colaborar de forma relevante para o desenvolvimento e aprimoramento de técnicas de amostragem do referido grupo faunístico.
\end{abstract}

Palavras-chave: Métodos; Inventário; Prática; Biodiversidade.

Please provide the English version of the title

This summary reports the results obtained in a practical activity of the discipline of methods for inventories of biodiversity offered by PPGCRENAC - IF GOIANO. After discussions in classrooms, methods for sampling medium and large mammals were chosen and applied in the field to record species that occur in the region. Three areas were studied and a wealth of 18 species was attributed to the region. Therefore, the methods were applied efficiently and the proposal of the discipline was fulfilled, contributing to the development and improvement of the sampling techniques of the referred fauna group.

Key words: Methods; Inventory; Practice; Biodiversity

\footnotetext{
Agradecimentos: Ao Prof. Dr. André Luís da Silva Castro por ter cedido ao grupo a câmera trap utilizada nas amostragens.

Para citar:

Quintão et al. (2020). Aplicação de métodos para amostragem de mamíferos de médio e grande porte. In: Anais do I Colóquio da Pós-Graduação do IF Goiano - Campus Urutaí, 4 a 5 de novembro de 2020 (p. 56). Urutaí GO: Multi-Science Journal, 3(3). DOI: https:// doi.org/10.33837/msj.v3i3.1349
} 


\title{
Aves de interesse conservacionista de um reservatório na região do médio rio Tocantins, Brasil
}

\author{
Karla Dayane de L. Pereira ${ }^{1^{*}}$, Arthur A. Bispo ${ }^{2}$, Daniel de Paiva Silva ${ }^{3}$ \\ ${ }_{1}$ Programa de Pós-Graduação em Conservação de Recursos Naturais do Cerrado (PPG-CRENAC), IF Goiano, Rod. Geraldo \\ Silva Nascimento KM 2,5 Zona Rural, Urutaí, GO, 75790-000, Brasil. ORCiD: https://orcid.org/0000-0003-1578-89480 \\ ${ }^{2}$ Laboratório de Etnobiologia e Biodiversidade, Universidade Federal de Goiás, Instituto Neotropical: Pesquisa e \\ Conservação, Goiânia, GO, 74690-970, Brasil. ORCiD: https://orcid.org/0000-0002-3375-1471 \\ ${ }_{3}^{3}$ Departamento de Ciências Biológicas, IF Goiano, Rodovia Geraldo Silva Nascimento KM 2,5 Zona Rural, Urutai, GO, \\ 75790-000, Brasil. ORCiD: https://orcid.org/0000-0002-2457-6245 \\ *E-mail:karla.biologia@yahoo.com.br
}

Os dados pontuais de ocorrência de espécies, obtidos muitas vezes por inventariamentos, são essências na elaboração de estratégias de conservação, principalmente de espécies de importância conservacionista, ou seja, aquelas ameaçadas de extinção ou regionalmente raras. Neste estudo são relatados registros de algumas espécies de aves ameaçadas e com distribuição pouco conhecida e/ou rara para os estados do Maranhão e Tocantins. Os resultados foram obtidos do programa de monitoramento de fauna da UHE Estreito (fase Pós-Enchimento). A avifauna foi monitorada durante 16 campanhas trimestrais ocorridas entre 2012 e 2015. Foram amostrados 12 pontos terrestres e três aquáticos, em quatro municípios na porção norte do estado do Tocantins e um município no sul do Maranhão. Para as informações de conservação das espécies, foram consultadas as Listas Vermelhas de Espécies Ameaçadas em nível Global (IUCN) e Nacional (ICMBio-MMA). Os dados de distribuição foram obtidos de literaturas científicas e bancos de dados Online. Foram registradas 15 espécies ameaçadas globalmente, sendo seis quase ameaçadas, oito vulneráveis e uma em perigo. Destas, duas espécies vulneráveis, Penelope ochrogaster Pelzeln, 1870 e Cercomacra ferdinandi Snethlage, 1928, também são consideradas vulneráveis em nível nacional. Além das espécies, $P$. ochrogaster e $C$. ferdinandi, foram identificadas mais seis espécies com distribuição rara ou pouco conhecida para o estado do Maranhão. As espécies, Asio flammeus (Pontoppidan, 1763) e Myiobius barbatus (Gmelin, 1789), merecem destaque pela raridade de ocorrência no Maranhão e no Tocantins. No total, foram registradas 21 aves de interesse conservacionista na região amostrada, destas, sete espécies são endêmicas do Brasil e/ou do Bioma Cerrado. A maioria das aves com algum grau de ameaça de extinção, encontram-se em declínio populacional devido à perda e alteração de seu habitat. Entre essas, $P$. ochrogaster e C. ferdinandi, são espécies que requerem uma maior atenção conservacionista nas áreas amostradas, pois além de estarem ameaçadas nacional e globalmente, elas possuem uma distribuição rara, cujos poucos registros conhecidos de ocorrência no Maranhão, estão restritos às áreas de influência do reservatório da UHE Estreito.

Palavras-chave: avifauna; distribuição; ocorrência; usina hidrelétrica.

Birds of conservation interest of a reservoir in the region of the middle Tocantins River, Brazil Here we identified some birds' species of threatened and with few known records for the states of Maranhão and Tocantins. The data resulted from environmental studies at the Estreito HPP, between 2012-2015. 21 birds of conservation interest were identified. Of these, 15 species have some degree of threat of extinction. Ten species were considered rare or little known for the state of Maranhão. Of these, two species were also considered little known for the state of Tocantins. Of all identified birds, two require more attention, as they are locally rare and are threatened globally and nationally.

Key words: birdlife; distribution; occurrence; hydroelectric power plant.

\footnotetext{
Agradecimentos: Ao Consórcio Estreito Energia (CESTE) pela autorização de uso dos dados. Aos técnicos, ajudantes e barqueiros envolvidos nos trabalhos de campo.

Para citar:

Pereira, K. D. L., Bispo, A. A., \& Silva, D. P. (2020). Aves de interesse conservacionista de um reservatório na região do médio rio Tocantins, Brasil. In: Anais do I Colóquio da Pós-Graduação do IF Goiano - Campus Urutaí, 4 a 5 de novembro de 2020 (p. 57). Urutaí - GO: Multi-Science Journal, 3(3). DOI:

https://doi.org/10.33837/msj.v3i3.1349
} 


\title{
Diversidade da avifauna em áreas de influência da Usina Hidrelétrica de Estreito, Brasil
}

\author{
Karla Dayane de L. Pereira ${ }^{1 *}$, Arthur A. Bispo ${ }^{2}$, Daniel de Paiva Silva ${ }^{3}$ \\ 1 Programa de Pós-Graduação em Conservação de Recursos Naturais do Cerrado (PPG-CRENAC), IF Goiano \\ ${ }^{2}$ Laboratório de Etnobiologia e Biodiversidade, Universidade Federal de Goiás, Instituto Neotropical: Pesquisa e Conservação \\ ${ }^{3}$ Departamento de Ciências Biológicas, IF Goiano, Urutaí, GO \\ *E-mail: karla.biologia@yahoo.com.br
}

\begin{abstract}
O licenciamento ambiental é um instrumento de políticas públicas ambientais que visa a gestão do desenvolvimento e do meio ambiente. Dessa forma, os estudos ambientais são importantes para se compreender as comunidades biológicas em áreas modificadas, ocasionadas pela implantação de empreendimentos de significativo impacto ambiental. Nesse sentido, o presente estudo objetivou identificar a diversidade avifaunística registrada nas áreas de influência da UHE Estreito. A usina está localizada em domínio do bioma Cerrado, na divisa dos estados do Tocantins e Maranhão, Brasil. Foi realizada uma revisão bibliográfica dos levantamentos obtidos das etapas de licenciamento ambiental desse empreendimento. As amostragens de campo contemplaram os 12 municípios atingidos pelo reservatório e foram divididas em três períodos: Estudos de Impacto Ambiental - EIA (três campanhas, ocorridas em 2001 e 2003), Monitoramentos fase préenchimento (nove campanhas; 2008 a 2010) e Pós-enchimento (16 campanhas; 2012 a 2015). As aves foram registradas por transectos, encontros ocasionais e captura por meio de neblina, esse último exceto durante o EIA. A lista revisada seguiu a ordem filogenética e nomenclatura proposta pela Lista das Aves do Brasil, organizada pelo CRBO/2015. A partir da revisão dos dados, foram desconsideradas oito espécies por possível identificação errônea em relação a distribuição, quatro obtidas exclusivamente por meio de entrevistas com moradores locais, e seis que tiveram sua identidade (ID) corrigida. Dessa forma, os estudos registraram 55.688 indivíduos de 429 espécies de aves, pertencentes a 72 famílias e 28 ordens. O pós-enchimento foi responsável pela maior diversidade registrada (riqueza $=358$ spp.; abundância $=36.659$ indivíduos), seguido do préenchimento (riqueza $=336$ spp.; abundância $=16.398$ indivíduos), e por último o EIA, com 314 espécies e 2.631 indivíduos. O pós-enchimento foi a etapa com o maior número de espécies exclusivas $(n=45)$. Durante todos os períodos de estudo da avifauna nas áreas de influência da UHE Estreito, foram identificadas espécies endêmicas, migratórias, raras e ameaçadas. No pósenchimento, foi registrada a espécie Catharus fuscescens (Stephens, 1817), uma ave migratória neártica, que foi documentada como primeiro registro para o estado do Maranhão. Os estudos ambientais podem não levar a um entendimento aprofundado da presença e/ou ausência, e da composição de uma comunidade. No entanto, estes estudos são necessários para se chegar à compreensão da diversidade, distribuição, estruturas e interações ecológicas das comunidades biológicas, subsidiando avaliações de impactos e caminhos para a conservação da biodiversidade.
\end{abstract}

Palavras-chave: aves; levantamento de fauna; licenciamento ambiental; reservatório.

Bird diversity in areas of influence of the Estreito Hydroelectric Power Plant, Brazil

Were reviewed the registered birds of the Estreito HPP, The data were obtained of the environmental studies carried out in the areas affected by the reservoir. In nine years of study, a significant diversity of the studied community was identified. In all phases of the studies, endemic, rare, threatened and migratory species were recorded. Even after filling the reservoir, the recorded bird diversity was superior to the other study phases. The results reinforce the importance of environmental licensing for the knowledge and understanding of biological communities and their relationship with the environment.

Key words: birds; fauna survey; environmental licensing; reservoir.

Agradecimentos: Ao Consórcio Estreito Energia (CESTE) pela autorização de uso dos dados. Aos técnicos, ajudantes e barqueiros envolvidos nos trabalhos de campo.

Para citar

Pereira, K. D. L., Bispo, A. A., \& Silva, D. P. (2020). Diversidade da avifauna em áreas de influência da Usina Hidrelétrica de Estreito, Brasil. In: Anais do I Colóquio da Pós-Graduação do IF Goiano - Campus Urutaí, 4 a 5 de novembro de 2020 (p. 58). Urutaí - GO: Multi-Science Journal, 3(3). DOI:

https://doi.org/10.33837/msj.v3i3.1349 


\title{
Efeitos dos nanoplásticos de poliestireno sobre Ctenopharyngodon idella (carpa capim) após exposição individual e combinada com nanopartículas de óxido de zinco
}

\author{
Fernanda Neves Estrela1, Abraão Tiago Batista Guimarães', Amanda Pereira da Costa \\ Araújo이 $^{2}$, Thiarlen Marinho da Luz ${ }^{3}$, Abner Marcelino Silva ${ }^{3}$, Guilherme Malafaia ${ }^{4}$ \\ ${ }^{1}$ Programa de Pós-Graduação em Biotecnologia e Biodiversidade, Universidade Federal de Goiás, Instituto de Patologia \\ Tropical e Saúde Pública, Goiânia, Brasil. \\ 2Programa de Pós-Graduação em Ciências Ambientais, Universidade Federal de Goiás - Campus Samambaia, Goiânia, Brasil. \\ ${ }^{3}$ Laboratório de Pesquisas Biológicas, Instituto Federal Goiano - Campus Urutaí, Urutaí, Brasil \\ ${ }^{4}$ Programa de Pós-Graduação em Conservação de Recursos Naturais do Cerrado, Instituto Federal Goiano - Campus Urutaí, \\ Urutaí, Brasil
}

\begin{abstract}
Apesar da toxicidade de muitos nanomateriais já ter sido demonstrada, os efeitos toxicológicos da exposição a alguns deles são pouco conhecidos em peixes de água doce. Esse é o caso dos nanoplásticos de poliestireno (PS NPs) e das nanopartículas de $\mathrm{ZnO}$ ( $\mathrm{ZnO} \mathrm{NPs}$ ) em concentrações ambientalmente relevantes. O objetivo do nosso estudo, foi avaliar os efeitos da PS NPs em peixes juvenis Ctenopharyngodon idella (carpa capim) individual e combinação com ZnO NPs, utilizando biomarcadores comportamentais. Após $72 \mathrm{~h}$ de exposição, os animais foram submetidos à uma bateria de testes, pressupondo que os nanopoluentes pudessem impactar o comportamento inato dos animais frente à distintos estímulos. O primeiro teste foi o campo aberto, o teste consistiu em introduzir o animal (individualmente) no centro da arena (com paredes opacas) e filmá-lo por 3 min para posterior registro da distância percorrida e velocidade de natação, bem o percentual de tempo de locomoção dos animais nos quadrantes centrais do aparato. Em seguida, realizou-se o teste do espelho, em nosso estudo, o teste consistiu em substituir o papel parede de um dos lados do aquário por um espelho posicionado em ângulo reto. Os animais foram monitorados por um período de $5 \mathrm{~min}$. Por fim realizamos o teste da substância alarme que consistiu em aclimatar os animais individualmente em uma arena circular de parede opaca $(\varnothing=15 \mathrm{~cm}$; altura da lâmina d'água $=3 \mathrm{~cm}$ ) por um período de $5 \mathrm{~min}$ e, em seguida, adicionar $1 \mathrm{~mL}$ de água purificada na região central, sendo os animais filmados por $3 \mathrm{~min}$. Posteriormente, $1 \mathrm{~mL}$ da solução contendo substâncias de alarme (preparadas previamente) foi adicionado no aparato e os animais avaliados por mais $3 \mathrm{~min}$. Os vídeos foram analisados no PlusMZ e todas as análises e elaborações gráficas foram realizadas no GraphPad Prism versão 7.00 (GraphPad Software, La Jolla California USA).Nossos dados indicam que mesmo em pequenas concentrações $(760 \mu \mathrm{g} / \mathrm{L})$ e por um curto período de exposição (3 dias), não foi observado déficit em locomoção, nem índices preditivos de ansiedade, já os PS NPs individualmente e em combinação com as ZnO NPs afetaram a resposta comportamental dos animais no teste do espelho. Notamos que todos os tratamentos induziram, equitativamente, a inatividade de C. idella frente a substâncias de alarme. Novos estudos precisam ser desenvolvidos para avaliação da (eco)toxicidade de misturas de poluentes envolvendo nanomateriais.
\end{abstract}

Palavras-chave: Ecotoxicologia; nanomateriais; toxicologia aquática; peixes.

Effects of polystyrene nanoplastics on Ctenopharyngodon idella (grass carp) after individual and combined exposure with zinc oxide nanoparticles

The toxicity of polystyrene nanoparticles (PS NPs) and ZnO nanoparticles ( $\mathrm{ZnO} \mathrm{NPs),} \mathrm{in} \mathrm{combination} \mathrm{is} \mathrm{poorly}$ known. Thus, the aim of the current study was to evaluate the effects of PS NPs $(760 \mu \mathrm{g} / \mathrm{L})$ on Ctenopharyngodon idella exposed to it, both in separate and in combination with $\mathrm{ZnO} N P s(760 \mu \mathrm{g} / \mathrm{L})$, based on behavioral. Current data have indicated that PS NPs, for a short exposure period ( 3 days), both in separate and in combination with nanoparticles, have affected animals' response to the mirror test. On the other hand, all treatments have equally induced C. idella inactivity towards alarm substances.

Key words: Ecotoxicology; Nanomaterials; Aquatic toxicology, Fish,; Mixtures of pollutants

Agradecimentos: Instituto Federal Goiano e o Laboratório de Pesquisas Biológicas

Para citar: Estrela, F. N. , Batista, A. G. T., Marinho, T. D. L., Silva, A. M., Araujo, A. P. D. C. Malafaia, G. (2020). Effects of polystyrene nanoplastics on Ctenopharyngodon idella (grass carp) after individual and combined exposure with zinc oxide nanoparticles. In: Anais do I Colóquio da Pós-Graduação do IF Goiano - Campus Urutaí, 4 a 5 de novembro de 2020 (p. 59). Urutaí - GO: Multi-Science Journal, 3(3). DOI: https://doi.org/10.33837/msj.v3i3.1349 